\title{
Validity of Generalized Boundary Conditions and Singular Integral Equation Method in the Scattering of Light by Thin Dielectric Strips
}

\author{
Olga V. Shapoval, \\ Laboratory of Micro and Nano-Optics \\ Institute of Radio-Physics and Electronics of National \\ Academy of Sciences of Ukraine \\ Proskury st. 12, Kharkiv 61085, Ukraine \\ olga.v.shapoval@gmail.com
}

\author{
Ilya O. Sukharevsky, Ayhan Altintas \\ Department of Electrical and Electronics Engineering \\ Bilkent University \\ Ankara, Turkey
}

\begin{abstract}
We consider the two-dimensional (2D) scattering of a plane wave of light by a thin flat dielectric nanostrip. Empirical method of generalized boundary conditions and singular integral equations on the strip median line is compared with Muller boundary integral equations method that does not assume the strip thickness to be small. The conclusions are achieved about the validity of approximate models for thin dielectric strips.
\end{abstract}

Keywords - thin strip, generalized boundary conditions, scattering, integral equations.

\section{INTRODUCTION}

Thin material strips are frequently met in the nano-optical technologies and applications because they are relatively easy manufactured with today's molecular-beam lithography and similar techniques. Still the accurate modeling and prediction of their electromagnetic behavior is a difficult computational task. This is because the strip-like nano-optical objects combine high values of dielectric permittivity and two different scales in their cross-sectional geometry. Their width can be larger than the wavelength while their thickness is only a fraction of it. This makes a burden for any existing commercial software and many in-house codes.

In [1], we have suggested an efficient numerical method for the analysis of 2D wave scattering by a thin material strip (see Fig. 1). Similarly to [2,3], it is based on the generalized boundary condition (GBC), which reduces the problem to two singular integral equations (SIE) on the strip median line. Further we use a meshless Nystrom-type discretization that provides guaranteed convergence and small size matrices. Thanks to this, we have computed, in [4], the scattering by finite gratings of hundreds of metal nanostrips. However, since the GBC is derived for an infinite thin material slab, its applicability for shorter and thicker finite strips, near the resonances, and at the grazing incidence can be questioned.

The domain of applicability of the GBC-SIE approach can be established only by confronting its numerical results with the reference ones. As such a trusted numerical solution, sufficiently accurate data obtained with independent method must be used. This method must take full account of strip thickness and have mathematically guaranteed convergence, which entails a control of accuracy, to machine precision, by taking larger the discretization order.

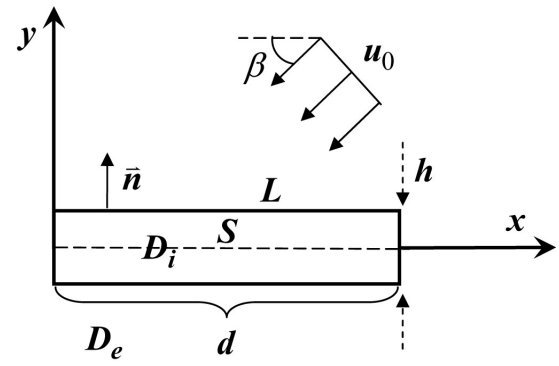

Fig. 1. Cross-sectional view of a free-standing thin dielectric strip of relative permittivity $\varepsilon$, width $d$ and thickness $h$, illuminated by a plane wave incident under the angle $\beta$.

The boundary IE (BIE) techniques are one of the most popular computational tools in the scattering of waves by 2-D homogeneous dielectric bodies, being more economic than volume IE [5]. Here, certain cautiousness must be a rule because many forms of BIE are corrupted by the presence of spurious real-valued eigenvalues [6]. This drawback can be avoided if one uses the Muller boundary IE (MBIE) that is a pair of coupled second-kind IE for the field tangential components [7-10]. Additionally, MBIE is a Fredholm secondkind IE, and therefore the convergence of its discretizations is guaranteed by the Fredholm theorems.

Several numerical schemes for solving MBIE are known that guarantee convergence. The most economic one is the Nystrom-type meshless discretization using interpolation polynomials and quadrature formulas. The schemes, which have been devised so far are all related to the smooth closed contours in 2-D [8,9]. However this treatment must be modified if the contour is only piece-wise smooth [11].

\section{Problem STATEMENT}

Consider an electromagnetic plane wave incident normally at the angle $\beta$ on a flat dielectric strip (infinite along the $z$ axis), see Fig. 1. The strip $D_{i}=\{(x, y): 0 \leq x \leq d,|y| \leq h / 2\}$ 


\section{IEEE XXXIV International Scientific Conference Electronics and Nanotechnology (ELNANO)}

has complex relative permittivity $\varepsilon$, width $d$, thickness $h$, and cross-section contour $L$. The outer medium is free space.

Assume that a plane-wave is incident. Its $z$-component of the $\mathrm{E}(\mathrm{H})$ field is given as $U^{i n c}(\vec{r})=e^{-i k_{0}(x \cos \beta+y \sin \beta)}$, where $\vec{r}=(x, y), k_{0}=\omega / c$, and $c$ is the free-space light velocity. In the presence of strip, the total field $U(\vec{r})$ is a sum of this function and the scattered field, $U^{s c}(\vec{r})$. The latter function must satisfy relevant Helmholtz equation inside and outside the strip and the continuity of tangential components across $L$. For uniqueness, $U^{s c}(\vec{r})$ must also satisfy Sommerfeld radiation condition and condition of local energy finiteness.

\section{Muller Boundary INTEGRAL EQUATIONS METHOD}

MBIE are derived using the manipulations with Green's formulas and theorems of the potential theory [12] - see [10]. In the $2 \mathrm{D}$ case, such derivation can be found in $[7,8]$, so that the resulting equations have the following form:

$$
\begin{aligned}
U(\vec{r})+\int_{L} K_{11}\left(\vec{r}, \vec{r}^{\prime}\right) U\left(\vec{r}^{\prime}\right) d \vec{r}^{\prime}-\int_{L} K_{12}\left(\vec{r}, \vec{r}^{\prime}\right) V\left(\vec{r}^{\prime}\right) d \vec{r}^{\prime}=U^{i n c}(\vec{r}) \\
\\
\frac{1+p}{2} V(\vec{r})+\int_{L} K_{21}\left(\vec{r}, \vec{r}^{\prime}\right) U\left(\vec{r}^{\prime}\right) d \vec{r}^{\prime}- \\
-\int_{L} K_{22} V\left(\vec{r}^{\prime}\right) d \vec{r}^{\prime}=V^{i n c}(\vec{r}), \vec{r} \in L
\end{aligned}
$$

Here, $V(\vec{r})=\partial U(\vec{r}) / \partial n$ is the limit value of the normal derivative of the total field function on the closed contour $L$ of the scatterer from the inner side of it, the normal unit vector $\vec{n}$ is directed to the outer domain $D_{e}$ (free space), $d \vec{r}^{\prime}$ is the elementary arc length, and the constant is $p=1$ in the Epolarization case and $p=1 / \varepsilon$ in the H-polarization case.

The kernel functions of MBIE are given by

$$
\begin{aligned}
& K_{11}=\frac{\partial G_{i}}{\partial n^{\prime}}-\frac{\partial G_{e}}{\partial n^{\prime}}, \quad K_{12}=G_{i}-p G_{e}, \\
& K_{21}=\frac{\partial^{2} G_{i}}{\partial n \partial n^{\prime}}-\frac{\partial^{2} G_{e}}{\partial n \partial n^{\prime}}, \quad K_{22}=\frac{\partial G_{i}}{\partial n}-p \frac{\partial G_{e}}{\partial n}
\end{aligned}
$$

where $G_{(i, e)}=G_{(i, e)}\left(\vec{r}, \vec{r}^{\prime}\right)=(i / 4) H_{0}^{(1)}\left(k_{i, e} \rho\right)$ are the Green's functions of the homogeneous media with the wavenumbers $k_{e}=k_{0}, k_{i}=k_{0} \sqrt{\varepsilon}$, and $\rho=\left|\vec{r}-\vec{r}^{\prime}\right|$.

As known [7,8], if $L$ is a Lyapunov surface then the kernel functions have only logarithmic singularities, and (1), (2) together are a Fredholm second kind IE. This guarantees convergence of conventional moment-method schemes of its discretization. However, the assumption of $L$ to be a Lyapunov surface is essential here, since in non-regular points of contour the unit normal vector and hence field components and their derivatives are not defined. Traditional way to overcome this problem is the approximation of irregular curve with a smooth one and then application of quadratures for the $2 \pi$-periodic functions [8].
We will follow a different approach. Namely, we subdivide the strip contour $L$ into four straight intervals, extract logarithmic singularities from (3) and (4), and apply quadratures on each smooth segment. The discretization performed in this manner is not sensitive to the irregularities of the contour provided that the interpolation nodes do not coincide with edge points. After introducing new continuous kernels (with the extracted singularities $[7,8]$ ), we divide the segments of $L$ into $N$ sub-sections, and assume that unknown functions are constants at each sub-section. Numerical integration based on the rectangle rule leads to a matrix equation with respect to the unknowns. To reach the accuracy of the order of $10^{-4}$ in the far field, we have taken, in computations, $N=600$. Verification of solution has been performed by comparing the results with [5].

\section{GENERALIZEd Boundary CONDITIONS - Singular INTEGRAL EQUATION METHOD}

GBC-SIE is an empirical method, in the sense that it does not follow directly from MBIE in mathematically justified manner; instead it uses physical assumptions for thin strips.

The essence of these assumptions is as follows: if $h<<\lambda$ ( $\left.k_{0} h<<1\right)$, one can shrink the strip cross-section $D_{i}$ to its median line, $S=\{(x, 0): x \in[0, d], y=0\}$, exclude the field inside the strip from consideration, and impose, at $S$, two-side GBC originally derived in for an infinite thin material slab illuminated by a plane wave [13-18]. We will use the following form of GBC adopted in [3] as more transparent:

$$
\begin{gathered}
(1 / 2)\left[\vec{E}_{T}^{+}(x, 0)+\vec{E}_{T}^{-}(x, 0)\right]=R Z_{0} \vec{n} \times\left[\vec{H}_{T}^{+}(x, 0)-\vec{H}_{T}^{-}(x, 0)\right], \\
(1 / 2)\left[\vec{H}_{T}^{+}(x, 0)+\vec{H}_{T}^{-}(x, 0)\right]=-Q Z_{0}^{-1} \vec{n} \times\left[\vec{E}_{T}^{+}(x, 0)-\vec{E}_{T}^{-}(x, 0)\right],
\end{gathered}
$$

Here, $\vec{n}=(0,1)$ is the unit normal vector, $Z_{0}$ is the freespace impedance, the indices \pm correspond to the field limit values at the top and bottom sides of the strip, respectively. Equations (9) are the Ohm's law for the effective surface currents. Therefore the coefficients $R$ and $Q$ are called the electric and magnetic resistivities. They have several forms depending on the slab dielectric permittivity. If the contrast is high, $|\varepsilon| \gg>1$, then $[13,14,16,3]$

$$
R=\frac{i}{2 \varepsilon^{1 / 2}} \cot \left(\frac{k_{0} h \varepsilon^{1 / 2}}{2}\right), Q=\frac{i \varepsilon^{1 / 2}}{2} \cot \left(\frac{k_{0} h \varepsilon^{1 / 2}}{2}\right)
$$

If, alternatively, the contrast is low, so that $k_{0} h|\varepsilon-1| \ll 1$, then [15]

$$
R=i\left[\varepsilon^{1 / 2}(\varepsilon-1) k_{0} h\right]^{-1}, Q=i \varepsilon^{1 / 2}\left[(\varepsilon-1) k_{0} h\right]^{-1}
$$

A modified version of (10) has been proposed in [17]. It keeps validity for $\varepsilon \rightarrow 1$ by taking into account the phase compensation for the reduction of the slab area to the median line of zero thickness. This entails

$$
R^{*}=\frac{\vartheta-R-\vartheta^{2} R}{4 \vartheta R-\vartheta^{2}-1}, \quad Q^{*}=\frac{\vartheta-Q-\vartheta^{2} Q}{4 \vartheta Q-\vartheta^{2}-1}
$$

where $\vartheta=i \cot \left(k_{0} h / 4\right)$, and $R$ and $Q$ are the same as in (10). 


\section{IEEE XXXIV International Scientific Conference Electronics and Nanotechnology (ELNANO)}

When GBC (9) is imposed at a finite strip, it must be supplemented with the condition of local finiteness of power and the radiation condition. The field singularities at the edges of a resistive strip have been analyzed in [19]; they are different from the right-angle dielectric wedge.

Although GBC (9) does not depend on the width $d$, it is natural to expect that for very narrow strips, say $d<\lambda$, its use is too far from infinite-slab situation.

Derivation of SIE is based on the use of the potential theory [12]. We seek the scattered field as a sum of singlelayer and double-layer potentials,

$$
U^{s c}(\vec{r})=k_{0} \int_{S} v\left(\vec{r}^{\prime}\right) G\left(\vec{r}, \vec{r}^{\prime}\right) d \vec{r}^{\prime}+\int_{S} w\left(\vec{r}^{\prime}\right) \frac{\partial G\left(\vec{r}, \vec{r}^{\prime}\right)}{\partial \vec{r}^{\prime}} d \vec{r}^{\prime},
$$

where $U(\vec{r})$ is either $H_{z}(\vec{r})$ or $E_{z}(\vec{r})$ depending on the polarization, and $G\left(\vec{r}, \vec{r}^{\prime}\right)$ is the free-space Green's function. From the properties of the limit values of potentials it follows that unknown functions are $v(\vec{r})=\partial / \partial \vec{n}(\vec{r})\left[U^{+}(\vec{r})-U^{-}(\vec{r})\right]$ and $w(\vec{r})=U^{+}(\vec{r})-U^{-}(\vec{r})$.

Using (13) in GBC (9) yields two independent IEs of the second kind with the same integration domain. In the $\mathrm{H}$ polarization case, on using the strip parameterization as $x=x(t)=d(t+1) / 2, y=y(t) \equiv 0$ for $t \in[-1,1]$ and new smooth unknown function $\tilde{w}(t)=w(t)\left(1-t^{2}\right)^{-1 / 2}$, we obtain

$$
\begin{gathered}
4 Q v\left(t_{0}\right)+\kappa \int_{-1}^{1} v(t) H_{0}^{(1)}\left(\kappa\left|t-t_{0}\right|\right) d t=f_{v}\left(\kappa, t_{0}\right) \\
4 R \tilde{w}\left(t_{0}\right) \sqrt{1-t_{0}^{2}}+\int_{-1}^{1} \tilde{w}(t) \sqrt{1-t^{2}} \frac{H_{1}^{(1)}\left(\kappa\left|t-t_{0}\right|\right)}{\left|t-t_{0}\right|} d t=f_{w}\left(\kappa, t_{0}\right)
\end{gathered}
$$

In the case of an E-polarized wave, the quantities $R$ and $Q$ exchange places. Here, $\kappa=k_{0} d / 2$ and the right-hand-part terms $f_{v}(\kappa, t)=4 i e^{-i \kappa(t+1) \cos \beta}$ and $f_{w}(\kappa, t)=4 \sin \beta e^{-i \kappa(t+1) \cos \beta}$ are defined by the incident wave.

Further we discretize SIEs (14) and (15) using a Nystromtype method, i.e. approximating the unknowns by polynomials and using quadrature rules of interpolation type of the order $N$ $[1,4]$. The theorems on quadratures ensure convergence of such numerical scheme with the rate of at least $1 / N$ if $N \rightarrow \infty$. This enables on to study not only the scattering by single strips but also by the finite and infinite gratings of them [20-23]. All data presented below have been computed with $10^{-4}$ accuracy that was achieved with $N=50$.

\section{Numerical Results}

To study validity and limitations of the GBC-SIE approach, we have compared its results with those of MBIE. We have considered both cases of the $\mathrm{E}$ and H-polarization.

\section{- E-polarization}

As a quantity to be compared, we have selected the total scattering cross-section (TSCS), whose definition can be found in [1]. Note that this is a far-field quantity and therefore it is not sensitive to the field behavior at the strip edges. In Fig. 2, we compare spectra of TSCS for a lossless strip with $\varepsilon=20$ and thickness $h / d=0.01$ under the broadside illumination $(\beta=\pi / 2)$.

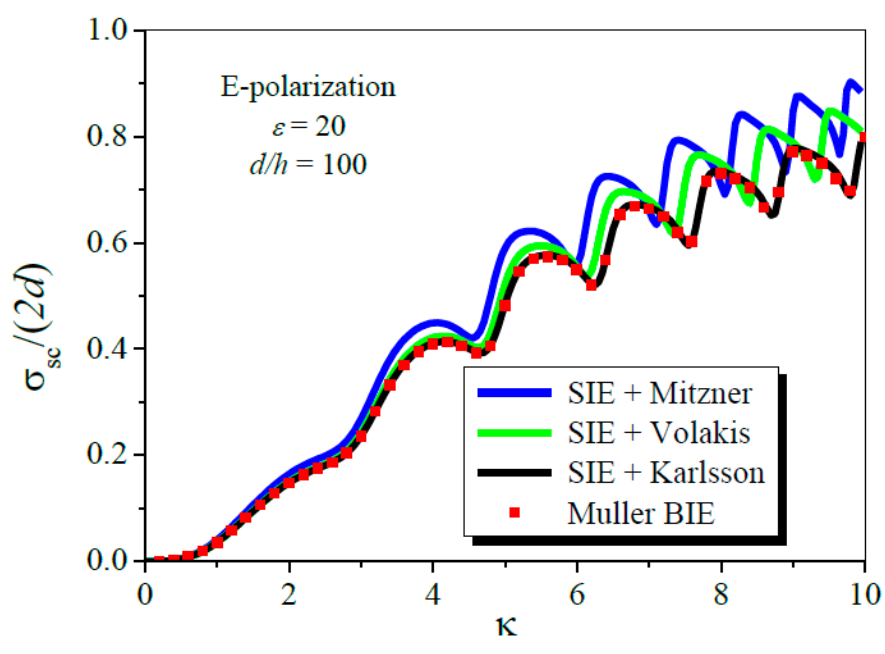

Fig. 2. Normalized RCS versus normalized frequency for a dielectric strip of $\varepsilon=20$ and thickness $h / d=00.1$ under the normal $(\beta=\pi / 2)$ incidence of the E-polarized plane wave.

As visible, the compensated model of (12) is the best. All models reproduce periodic Fabry-Perot resonances on the principal E-polarized surface wave of the corresponding slab. Still the largest errors are near these resonances because of the shifts of resonance frequencies.

The relative errors versus varying frequency are shown in Fig. 3 where MBIE results are taken as a reference, so that

$$
\text { error }=\left|\sigma_{s c}^{G B C-S I E}-\sigma_{s c}^{M B I E}\right| / \sigma_{s c}^{M B I E}
$$

One can see that the error in Karlsson's model does not exceed $2 \%$ in contrast to $12 \%$ for the model of (10). The lowcontrast model of (11) is good only for very thin strips: it can be used with a $2 \%$ or better accuracy if $\kappa<2.5$, i.e. where $k_{0} h|\varepsilon-1|<0.5$.

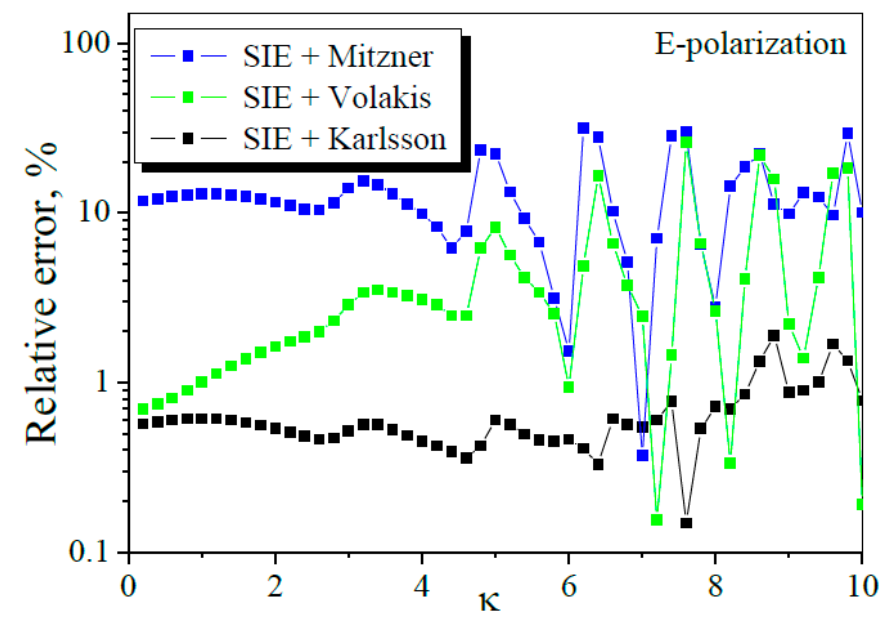

Fig. 3. Relative errors as a function of the normalized frequency for the same configuration as in Fig. 2. 


\section{IEEE XXXIV International Scientific Conference Electronics and Nanotechnology (ELNANO)}

\section{- H-polarization}

In the H-case the compensated high-contrast model of (12) also agrees very well with the MBIE reference data (see Fig. 4). The corresponding errors are shown in Fig. 5: the models of (12) and (11) provide $2 \%$ and $10 \%$ accuracy, respectively.

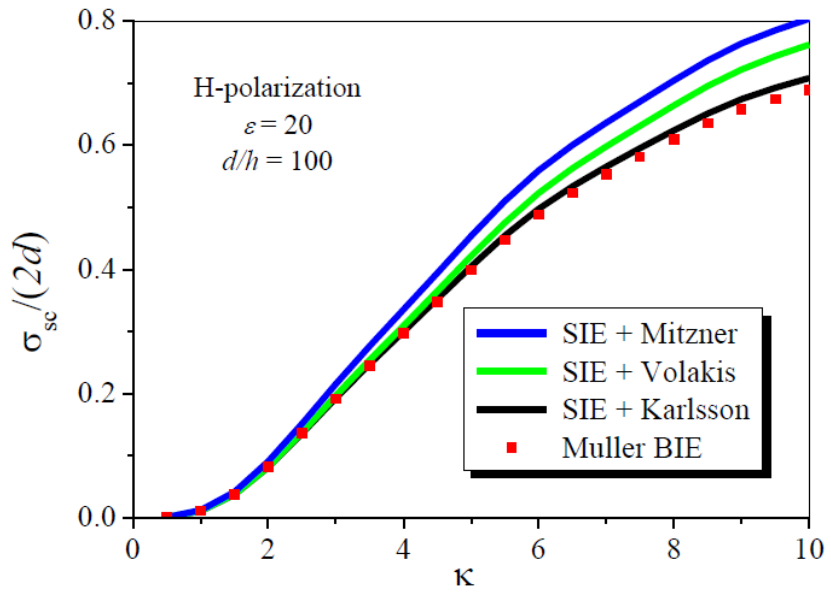

Fig. 4. The same as in Fig. 2 however for the H-polarization case.

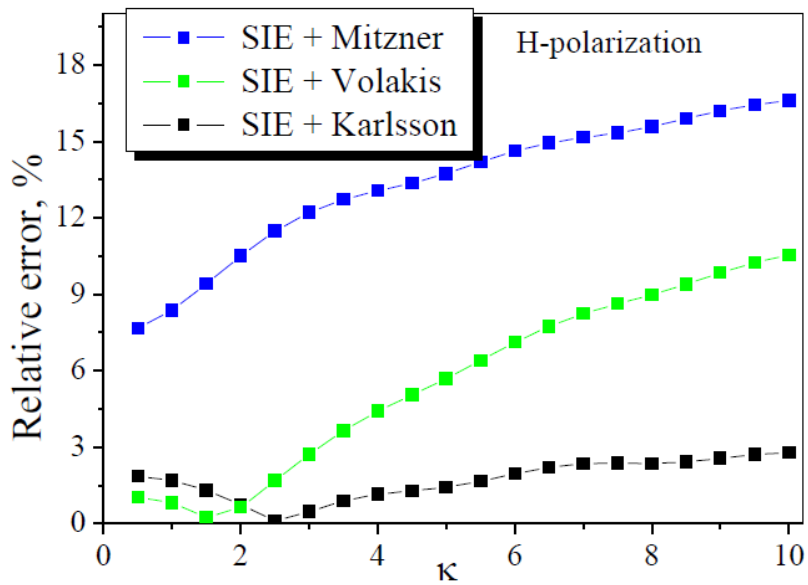

Fig. 5. The same as in Fig. 3 however for the H-polarization case.

\section{CONCLUSIONS}

We have compared two approaches to the 2D scattering of light by dielectric nano strips: approximate model based on the GBC and SIE with a Nystrom-type discretization and accurate model based on the Muller boundary IE discretized with another Nystrom method. Both the E- and H-polarization cases have been considered. As any empirical technique, GBC-SIE approach has certain limitations. Nevertheless in either polarization, the compensated high-contrast model of [17], together with GBC and SIE, is able to provide accuracy of $2 \%$ both in and off the resonances.

\section{REFERENCES}

[1] O. V. Shapoval, R. Sauleau, and A. I. Nosich, "Scattering and absorption of waves by flat material strips analyzed using generalized boundary conditions and Nystrom-type algorithm," IEEE Trans. Antennas Propag., vol. 59, no 9, pp. 3339-3346, 2011.
[2] T. B. A. Senior and J. L. Volakis, "Sheet simulation of a thin dielectric layer," Radio Sci., vol. 22, no. 7, pp. 1261-1272, 1987.

[3] E. Bleszynski, M. Bleszynski, and T. Jaroszewicz, "Surface-integral equations for electromagnetic scattering from impenetrable and penetrable sheets," IEEE Antennas Propag. Mag., vol. 36, pp. 14-25, 1993.

[4] O. V. Shapoval, R. Sauleau, and A. I. Nosich, "Modeling of plasmon resonances of multiple flat noble-metal nanostrips with a median-line integral equation technique," IEEE Trans. Nanotechnology, vol. 12, no 3, pp. 442-449, 2013.

[5] J. H. Richmond, "Scattering by a thin dielectric strip," IEEE Trans. Antennas Propag., vol. AP-33, no 1, pp. 64-68, 1985.

[6] A. F. Peterson, "The 'interior resonance' problem associated with surface integral equations of electromagnetics: numerical consequences and a survey of remedies," Electromagnetics, vol. 10, pp. 293-312, 1990.

[7] S.V. Boriskina, T.M. Benson, P. Sewell, A.I. Nosich, "Spectral shift and Q-change of circular and square-shaped optical microcavity modes due to periodic sidewall surface roughness", J. Optical Society of America B, vol. 21, no 10, pp. 1792-1796, 2004.

[8] E. I. Smotrova, V. Tsvirkun, I. Gozhyk, C. Lafargue, C. Ulysse, M. Lebental, and A. I. Nosich, "Spectra, thresholds and modal fields of a kite-shaped microcavity laser," J. Optical Society America B, vol. 40, no 6, pp. 1732-1742, 2013.

[9] J. L. Tsalamengas, "Exponentially converging Nystrom method in oblique diffraction of arbitrarily polarized waves by bianisotropic/chiral cylinders with arbitrary smooth cross section," IEEE Trans. Antennas Propag., vol. 61, no 7, pp. 3362-3673, 2013.

[10] C. Muller, Foundations of the Mathematical Theory of Electromagnetic Waves, Berlin, Springer, 1969 (German edition, 1957).

[11] J. Helsing and A. Karlsson, "An accurate boundary value problem solver applied to scattering from cylinders with corners," IEEE Trans. Antennas Propag., vol. 61, no 7, pp. 3693-3700, 2013.

[12] N.M. Gunter, Potential Theory and its Applications to Basic Problems of Mathematical Physics, Frederick Ungar Publ., New York, 1967.

[13] K. M. Mitzner, "Effective boundary conditions for reflection and transmission by an absorbing shell of arbitrary shape," IEEE Trans. Antennas Propag., vol. 16, pp. 706-712, 1968.

[14] R. F. Harrington and J. R. Mautz, "An impedance sheet approximation for thin dielectric shells," IEEE Trans. Antennas Propag., vol. 36, pp. 531-534, 1975 (in fact, this paper deals with the resistive sheet).

[15] J. L. Volakis, "High-frequency scattering by a thin material half-plane and strip," Radio Science, vol. 23, no 3, pp. 450-462, 1988.

[16] G. Bouchitté, "Analyse limite de la diffraction d'ondes électromagnétiques par une structure mince", C.R. Acad. Paris, t. 311, pp. 51-56, 1990.

[17] A. Karlsson, "Approximate boundary conditions for thin structures," IEEE Trans. Antennas Propag., vol. 57, no 1, pp. 144-148, 2009.

[18] Z. Nazarchuk and K. Kobayashi, "Mathematical modelling of electromagnetic scattering from a thin penetrable target," Progress in Electromag. Research, vol. 55, pp. 95-116, 2005.

[19] I. M. Braver, P. S. Fridberg, K. L. Garb, and I. M. Yakover, "The behavior of the electromagnetic field near the edge of a resistive halfplane," IEEE Trans. Antennas Propag., vol. 36, pp. 1760-1768, 1988.

[20] T. L. Zinenko, A. I. Nosich, and Y. Okuno, "Plane wave scattering and absorption by resistive-strip and dielectric-strip periodic gratings," IEEE Trans. Antennas Propagat., vol. 46, pp. 1498-1505, 1998.

[21] T.L. Zinenko, A.I. Nosich, "Plane wave scattering and absorption by flat gratings of impedance strips," IEEE Trans. Antennas Propagat., vol. 54, no 7, pp. 2088-2095, 2006.

[22] T.L. Zinenko, M. Marciniak, and A.I. Nosich, "Accurate analysis of light scattering and absorption by an infinite flat grating of thin silver nanostrips in free space using the method of analytical regularization," IEEE J. Sel. Top. Quant. Electron., vol. 19, no 3, art. 9000108/8, 2013.

[23] O.V. Shapoval and A.I. Nosich, "Finite gratings of many thin silver anostrips: optical resonances and role of periodicity," AIP Advances, vol. 3, no 4, pp. 042120/13, 2013. 